\title{
Successful Treatment of Histiocytic Sarcoma and Concurrent HIV Infection Using a Combination of CHOP and Antiretroviral Therapy
}

\author{
Kosuke Narita ${ }^{1}$, Rintaro Noro ${ }^{1}$, Masahiro Seike ${ }^{1}$, Masaru Matsumoto ${ }^{1}$, Kazue Fujita ${ }^{1}$, \\ Jiro Matsumura ${ }^{2}$, Mikiko Takahashi ${ }^{3}$, Masashi Kawamoto ${ }^{4}$ and Akihiko Gemma ${ }^{1}$
}

\begin{abstract}
Histiocytic sarcoma (HS) is a rare malignancy of soft tissues with an unknown etiology. The CHOP (cyclophosphamide, vincristine sulfate, doxorubicin hydrochloride and prednisolone) regimen is often adopted as first-line chemotherapy; however, its therapeutic efficacy against HS is usually low. We herein first present the case of a patient with HS who was infected with human immunodeficiency virus-1 (HIV) in whom treatment with a combination of CHOP and antiretroviral therapy (ART) was successful. The patient has been in complete remission for 12 months following the discontinuation of chemotherapy under continuous ART. This case report may help to promote further investigation of both HS and HIV-related malignancy.
\end{abstract}

Key words: histiocytic sarcoma, CHOP, human immunodeficiency virus-1 (HIV), antiretroviral therapy (ART)

(Intern Med 52: 2805-2809, 2013)

(DOI: 10.2169/internalmedicine.52.0523)

\section{Introduction}

Histiocytic sarcoma (HS) is a rare neoplasm of soft tissues (1). Generally, the disease involves the intestinal tract, skin and soft tissues. HS can occur in individuals of all ages, from infants to the elderly. The gender predominance is unclear. HS patients with solitary lesions usually have systemic symptoms, such as fever and weight loss. The diagnostic criteria for HS are based on immunohistochemistry (1) and are characterized by histiocytic markers, including CD68, CD163 and lysozyme. However, the pathogenesis of this disease remains unclear. It may arise from pluripotent germ cells and hematological malignancies, including malignant lymphoma, leukemia and myelodysplasia (2-4). Combination chemotherapy, such as CHOP (cyclophosphamide, vincristine sulfate, doxorubicin hydrochloride and prednisolone), can be used as first-line chemotherapy in HS patients with advanced-stage disease $(5,6)$. However, the prognosis is unfavorable due to the poor response to chemotherapy.

Immunodeficiency is recognized to be a risk factor for various tumors in HIV-infected patients (7). For example, the frequency of Kaposi's sarcoma, non-Hodgkin lymphoma and liver cancer is higher among HIV-positive patients than among HIV-negative patients. On the other hand, some malignancies are considered to be unrelated to HIV, including prostate, lung and colorectal cancers $(8,9)$. HS has not yet been reported in HIV-infected patients.

We herein present the first case of HS in an HIV-positive patient who was successfully treated with a combination of CHOP and antiretroviral therapy (ART).

\section{Case Report}

A 61-year-old man presented with a two-month history of a dry cough and generalized fatigue. The patient had a good performance status [Eastern Cooperative Oncology Group Performance Status Scale (ECOG PS) 0]. On a physical ex-

\footnotetext{
${ }^{1}$ Department of Pulmonary Medicine and Oncology, Graduate School of Medicine, Nippon Medical School, Japan, ${ }^{2}$ Department of Microbiology and Immunology, Nippon Medical School, Japan, ${ }^{3}$ Department of Analytic Human Pathology, Nippon Medical School, Japan and ${ }^{4}$ Department of Clinical Pathology, University Hospital, Mizonokuchi, Teikyo University School of Medicine, Japan

Received for publication March 5, 2013; Accepted for publication August 7, 2013

Correspondence to Dr. Masahiro Seike, mseike@nms.ac.jp
} 

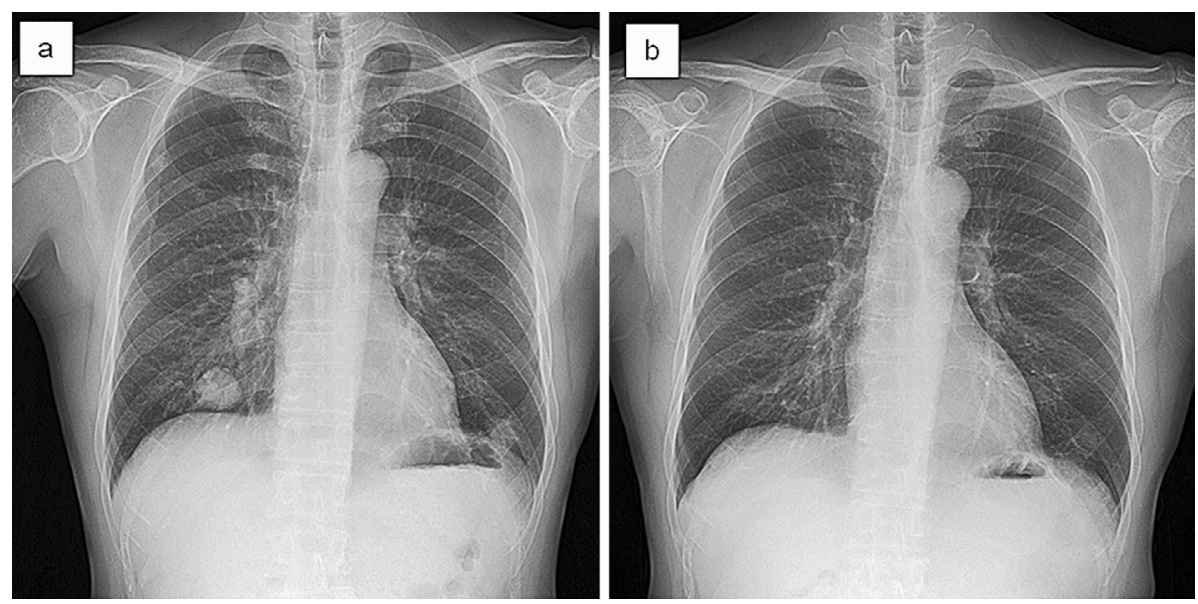

Figure 1. Chest radiography before and after treatment with CHOP and ART. (a) Multiple tumors were confirmed in both lung fields before treatment. (b) The bilateral lung lesions were completely diminished after treatment with CHOP and ART.
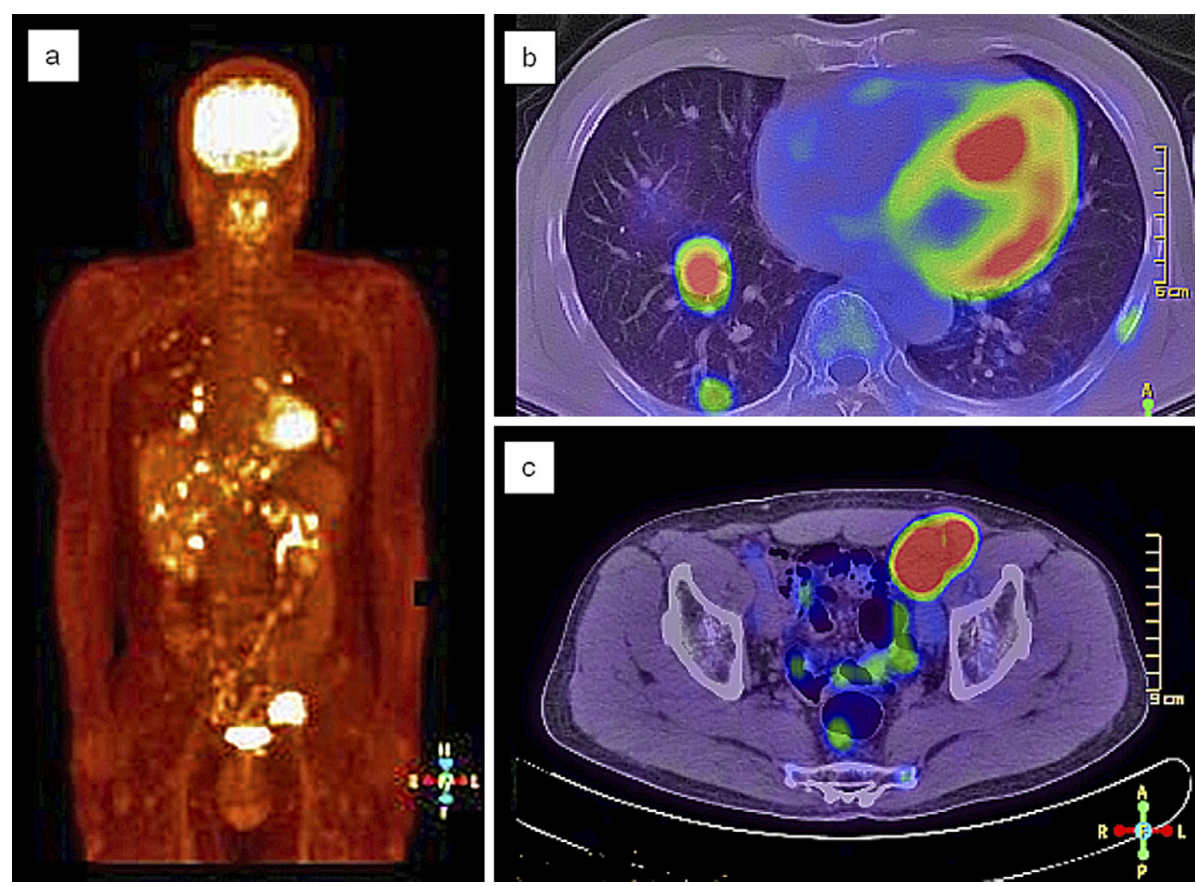

Figure 2. PET-CT before treatment with CHOP and ART. (a) A whole-body scan revealed multiple tumors with accumulation of ${ }^{18}$ F-FDG. (b) The lesions in the heart and lungs exhibited high uptake. (c) ${ }^{18}$ F-FDG was also intensely accumulated in the intra-abdominal mass.

amination, a subcutaneous tumor was found in the lower left abdomen. Chest radiography revealed multiple tumor lesions in both lung fields (Fig. 1a). Computed tomography (CT) showed multiple tumors distributed throughout the body (i.e., in the lungs, heart, liver, kidneys, adrenal glands, bone and intra-abdominal space). Positron emission tomography and computed tomography (PET-CT) demonstrated intense accumulation of fluorine-18 fluorodeoxyglucose $\left({ }^{18} \mathrm{~F}-\mathrm{FDG}\right)$ in the tumors, and the highest maximum standardized uptake value (SUVmax) was 12.3 in the tumor located in the heart (Fig. 2). The intra-abdominal tumor was $53 \times 31 \mathrm{~mm}$ in size and located adjacent to the left common iliac artery. A surgical biopsy of the intra-abdominal mass was performed.
Hematoxylin and eosin staining revealed infiltration by atypical multinucleated cells with abundant cytoplasm and inflammatory cells (Fig. 3a, b). Immunohistochemistry showed strong CD68 (DakoCytomation, Glostrup, Denmark; Fig. 3c) and S-100 (DakoCytomation, Glostrup, Denmark; Fig. 3d) staining, weak marginal positivity for CD1a (Beckman Coulter, CA, USA; Fig. 3e) and a lack of CD3 and CD20 (DakoCytomation, Glostrup, Denmark). Although no immunohistochemical evaluations of CD163 were performed, the CD68 expression was confirmed in the tumor cells, and the pathological findings fulfilled the diagnostic criteria for histiocytic sarcoma (1). A diagnosis of Kaposi's sarcoma was ruled out because immunohistochemistry using 


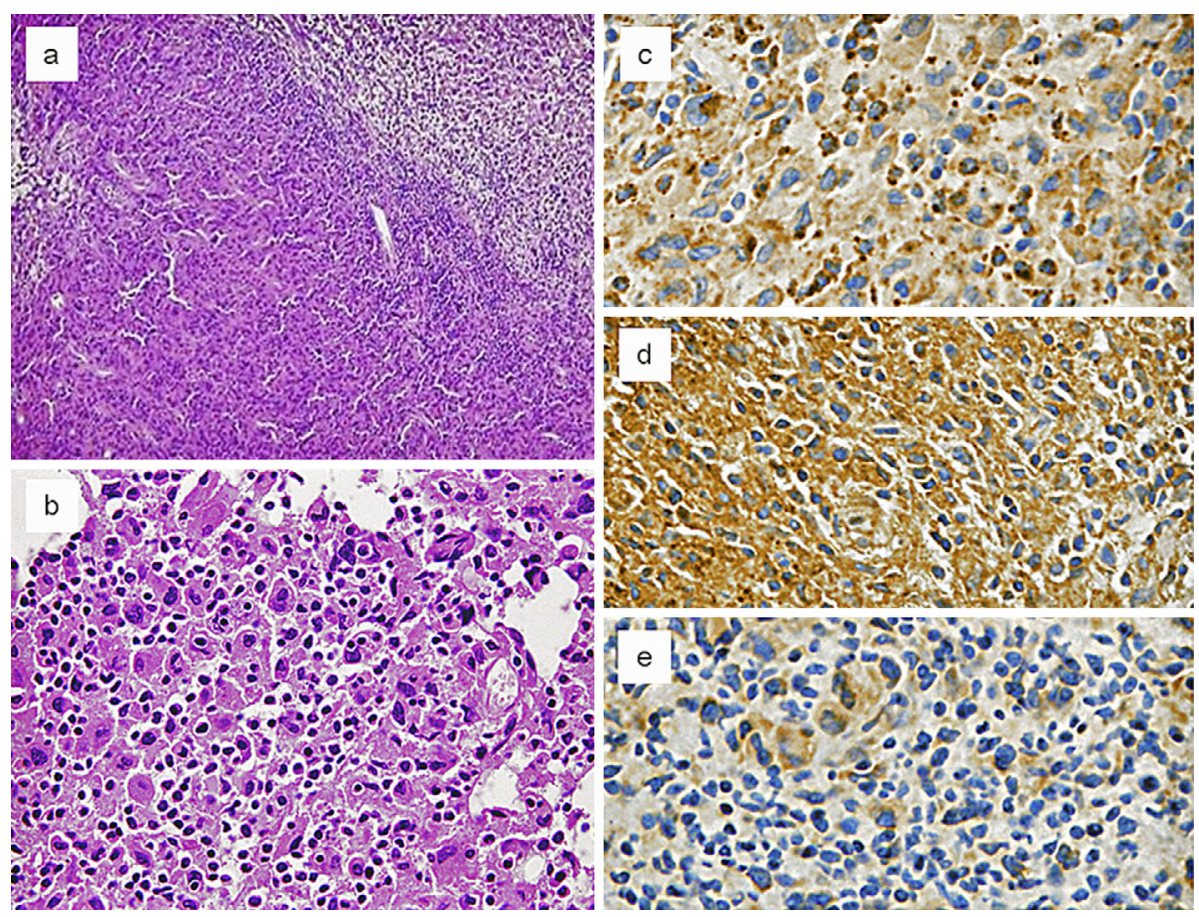

Figure 3. Surgical biopsy of the intra-abdominal mass. The biopsy specimen consisted of atypical multinucleated cells with abundant cytoplasm [Hematoxylin and Eosin staining; a: $\times 40$, b: $\times 400$ ]. Immunohistochemistry revealed that the tumor cells had strong positivity for CD68 (c) and S-100 (d) and weak marginal positivity for CD1a (e); each magnification: $\times 400$.
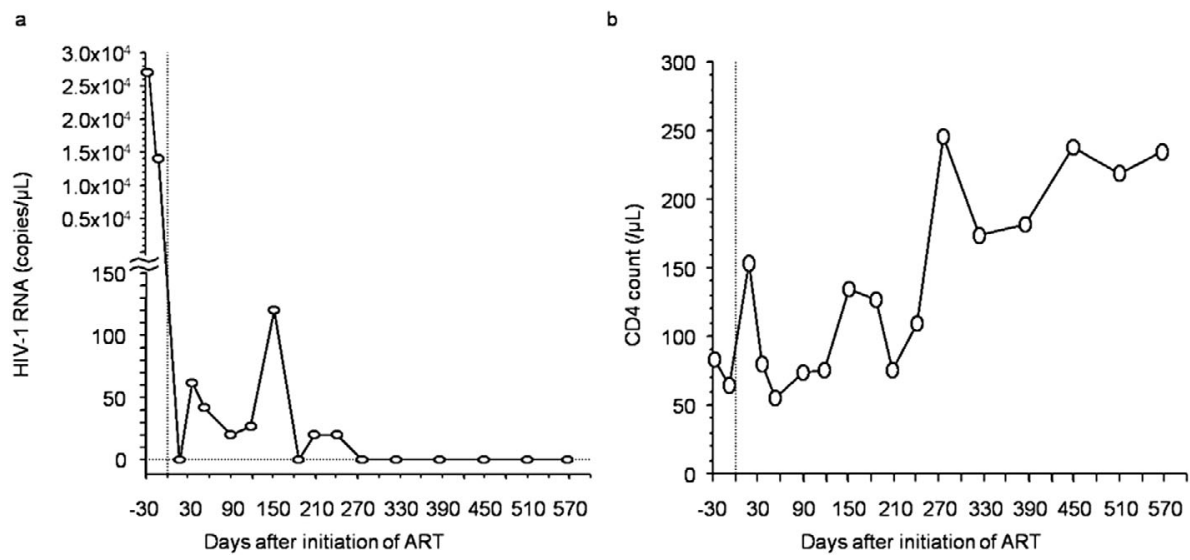

Figure 4. Clinical course of the HIV-1 RNA level and CD4 count. (a) The HIV-1 RNA level dramatically decreased after the initiation of ART. (b) The CD4 count increased progressively during ART.

anti-human herpes virus-8 (HHV-8) antibodies was negative in tumor samples obtained via a CT-guided biopsy of the liver metastases. One of the major differential diagnoses in this case was Langerhans cell sarcoma (LCS). LCS consistently expresses CD1a and S-100 (10). S-100 proteins were detected in our case. However, the tumor cells did not exhibit an expression of CD1a. Therefore, a diagnosis of LCS was ruled out, and the final pathological diagnosis was HS according to the WHO Classification of Tumors of Haematopoietic and Lymphoid Tissues 4th edition 2008 (1, 10). On the other hand, laboratory tests confirmed the presence of both HIV-1 antigens and antibodies, and the plasma HIV-1
RNA level was 27,000 copies/mL (Fig. 4a). The CD4positive lymphocyte count was only $83 / \mu \mathrm{L}$ (Fig. $4 \mathrm{~b}$ ). Moreover, neither HHV-8 DNA, cytomegalovirus antigens nor human T-cell leukemia virus-I (HTLV-I) antibodies were detected in the blood. There were also no abnormal findings in the blood chemistry or complete blood cell count, except for the decreased lymphocyte count. Therefore, the patient was diagnosed as having HS with HIV infection.

The patient was treated with ART (lamivudine $300 \mathrm{mg}$ / day, abacavir sulfate $600 \mathrm{mg} /$ day and raltegravir $800 \mathrm{mg} /$ day) for HIV because this regimen has the advantage of a relatively low frequency of adverse effects, such as unfavor- 


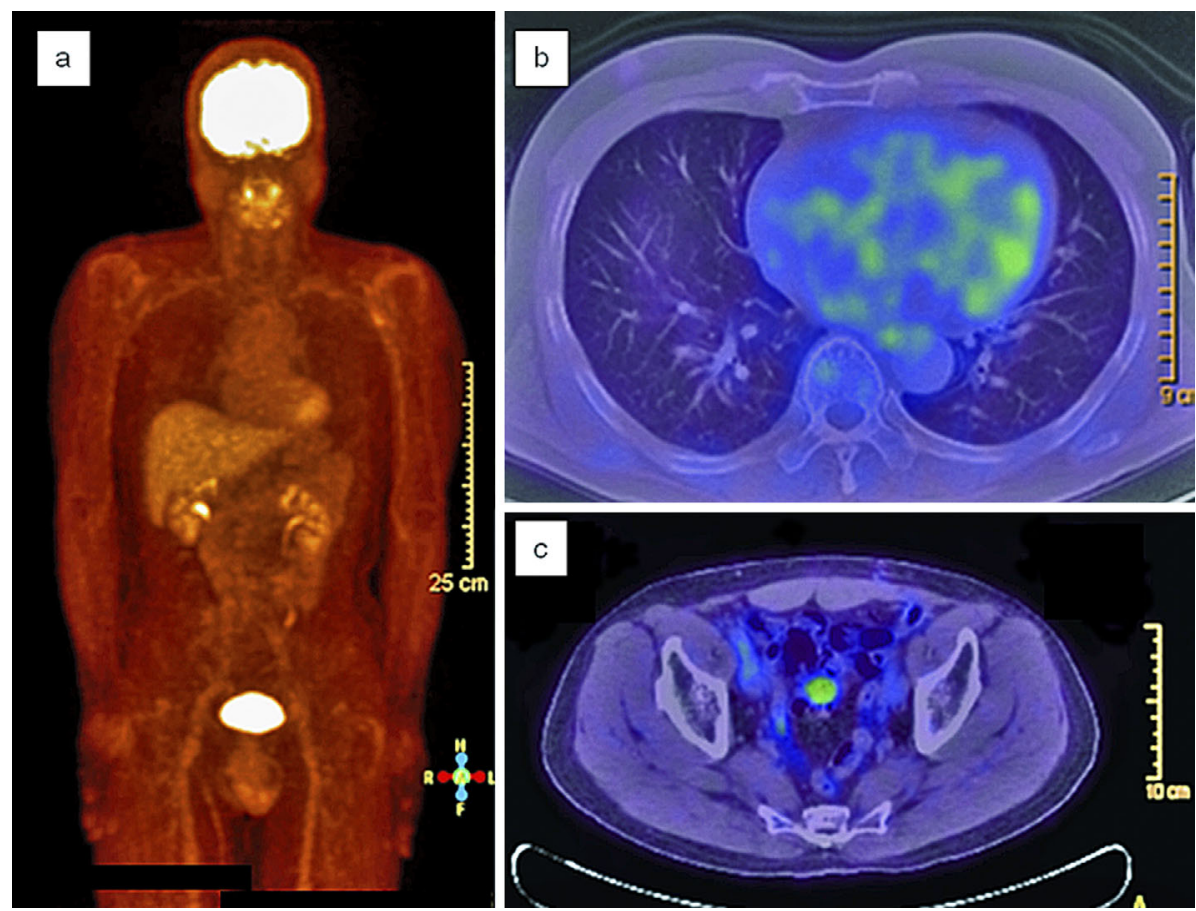

Figure 5. PET-CT after treatment with CHOP and ART. (a) A whole-body scan confirmed that there were no lesions with ${ }^{18}$ F-FDG uptake. (b) The tumors in the heart and lungs disappeared completely. (c) PET-CT also showed no recurrence of the intra-abdominal tumor.

able interactions with antitumor drugs (11). Trimethoprim/ sulfamethoxazole and fluconazole were administered to prevent Pneumocystis jirovecii pneumonia and candidiasis. In addition, six cycles of CHOP $\left(600 \mathrm{mg} / \mathrm{m}^{2}\right.$ of cyclophosphamide, $1.4 \mathrm{mg} / \mathrm{m}^{2}$ of vincristine sulfate and $50 \mathrm{mg} / \mathrm{m}^{2}$ of doxorubicin hydrochloride on day 1 with $100 \mathrm{mg} /$ day of oral prednisolone on days 1-5) were administered at 3-week intervals. The patient tolerated the entire course of chemotherapy well. Following the initiation of ART, the HIV viral load decreased dramatically, with a corresponding increase in the CD4 count (Fig. 4). Follow-up chest radiography and PET-CT showed dramatic decreases in the size of the pulmonary tumors and intra-abdominal mass after the six courses of CHOP (Fig. $1 \mathrm{~b}$ and Fig. 5). The patient was judged as having a complete response and has remained in remission for 12 months after the discontinuation of chemotherapy under continuous ART.

\section{Discussion}

To the best of our knowledge, there are no reports of HS occurring in patients with HIV infection. The etiology of HS remains unknown, and no relationships between HS and viral infections, including HIV, have been established (1). HIV infection is characterized by the progressive suppression of acquired immunity (i.e., acquired immunity deficiency syndrome, AIDS). HIV/AIDS patients are immunocompromised enough to suffer from opportunistic infectious diseases. In addition, HIV-infected patients are at greater risk of developing certain malignancies, such as Kaposi's sarcoma and non-Hodgkin lymphoma, that are known to be
HIV-associated malignancies (9). Immunodeficiency caused by HIV infection is thought to increase the incidence of HIV-associated malignancy (7). However, the role of HIV infection in the pathogenesis of malignant tumors is not fully understood.

In general, the prognosis of HS patients is poor. The median survival is reported to be $<12$ months $(5,12-17)$. Combination treatments, such as CHOP, can be used as first-line chemotherapy in advanced-stage HS patients $(5,6)$. However, HS patients often relapse after CHOP and may require additional therapies, such as second-line chemotherapy and autologous hematopoietic stem-cell transplantation $(6,18)$. The patient presented here was successfully treated with a combination of CHOP and ART. ART effectively decreased the level of HIV-1 RNA and increased the level of CD4 cells in the patient. The reduction in the HIV-1 viral load and the improvement in the CD4 count accomplished by ART are expected to result in better tolerance of chemotherapy and prolonged overall survival (19). ART may improve the immune response against HS and enhance the antitumor effects of CHOP.

In conclusion, to the best of our knowledge, this is the first report of the successful treatment of an HIV-infected HS patient using CHOP and ART. The pathogenesis of HS remains unknown; however, this case may help to further the investigation of both HS and HIV-related malignancies.

The authors state that they have no Conflict of Interest (COI).

\section{Acknowledgement}

The authors thank Dr. Tsunekazu Hishima (Department of Pa- 
thology, Tokyo Metropolitan Cancer and Infectious Diseases Center, Komagome Hospital, Tokyo, Japan) for performing the immunohistochemical analysis of HHV-8.

\section{References}

1. Grogan TM, Pileri SA, Chan JKC, et al. Histiocytic Sarcoma. In: WHO Classification of Tumours of Haematopoietic and Lymphoid Tissues. Swerdlow SH, Campo E, Harris NL, et al, Eds. IARC Press, Lyon, France, 2008: 356-357.

2. Feldman AL, Arber DA, Pittaluga S, et al. Clonally related follicular lymphomas and histiocytic/dendritic cell sarcomas: evidence for transdifferentiation of the follicular lymphoma clone. Blood 111: 5433-5439, 2008.

3. Feldman AL, Minniti C, Santi M, et al. Histiocytic sarcoma after acute lymphoblastic leukaemia: a common clonal origin. Lancet Oncol 5: 248-250, 2004.

4. Pileri SA, Grogan TM, Harris NL, et al. Tumours of histiocytes and accessory dendritic cells: an immunohistochemical approach to classification from the International Lymphoma Study Group based on 61 cases. Histopathology 41: 1-29, 2002.

5. Hornick JL, Jaffe ES, Fletcher CD. Extranodal histiocytic sarcoma: clinicopathologic analysis of 14 cases of a rare epithelioid malignancy. Am J Surg Pathol 28: 1133-1144, 2004.

6. Abu-Sanad A, Warsi A, Michel RP, et al. Long-term remission after autologous stem-cell transplantation for relapsed histiocytic sarcoma. Curr Oncol 19: e289-e291, 2012.

7. Carbone A, Gloghini A. AIDS-related lymphomas: from pathogenesis to pathology. Br J Haematol 130: 662-670, 2005.

8. Silverberg MJ, Chao C, Leyden WA, et al. HIV infection, immunodeficiency, viral replication, and the risk of cancer. Cancer Epidemiol Biomarkers Prev 20: 1-9, 2011.

9. Centers for Disease Control and Prevention. 1993 revised classification system for HIV infection and expanded surveillance case definition for AIDS among adolescents and adults. MMWR Re- comm Rep 41: 1-19, 1992.

10. Jaffe R, Weiss LM, Facchetti F. Tumors derived from Langerhans cells. In: WHO Classification of Tumours of Haematopoietic and Lymphoid Tissues. Swerdlow SH, Campo E, Harris NL, et al, Eds. IARC Press, Lyon, France, 2008: 358-360.

11. Rudek MA, Flexner C, Ambinder RF. Use of antineoplastic agents in patients with cancer who have HIV/AIDS. Lancet Onco 12: 905-912, 2011.

12. Pileri S, Mazza P, Rivano MT, et al. Malignant histiocytosis (true histiocytic lymphoma) clinicopathological study of 25 cases. Histopathology 9: 905-920, 1985.

13. Soria C, Orradre JL, García-Almagro D, et al. True histiocytic lymphoma (monocytic sarcoma). Am J Dermatopathol 14: 511517, 1992.

14. Miettinen M, Fletcher CD, Lasota J. True histiocytic lymphoma of small intestine. Analysis of two S-100 protein-positive cases with features of interdigitating reticulum cell sarcoma. Am J Clin Pathol 100: 285-292, 1993.

15. Lauritzen AF, Delsol G, Hansen NE, et al. Histiocytic sarcomas and monoblastic leukemias A clinical, histologic, and immunophenotypical study. Am J Clin Pathol 102: 45-54, 1994.

16. Cheuk W, Walford N, Lou J, et al. Primary histiocytic lymphoma of the central nervous system: a neoplasm frequently overshadowed by a prominent inflammatory component. Am J Surg Pathol 25: 1372-1379, 2001.

17. Vos JA, Abbondanzo SL, Barekman CL, et al. Histiocytic sarcoma: a study of five cases including the histiocyte marker CD 163. Mod Pathol 18: 693-704, 2005.

18. Gergis U, Dax H, Ritchie E, et al. Autologous hematopoietic stem-cell transplantation in combination with thalidomide as treatment for histiocytic sarcoma: a case report and review of the literature. J Clin Oncol 29: e251-e253, 2011.

19. Antinori A, Cingolani A, Alba L, et al. Better response to chemotherapy and prolonged survival in AIDS-related lymphomas responding to highly active antiretroviral therapy. AIDS 15: 14831491, 2001.

(C) 2013 The Japanese Society of Internal Medicine http://www.naika.or.jp/imonline/index.html 